\title{
Construction and evaluation of a novel tissue-engineered bone device
}

\author{
ZHENGQI CHANG $^{1 *}, \mathrm{JUNCHAO}^{\mathrm{XING}}{ }^{2 *}$ and XIUCHUN YU ${ }^{1}$ \\ ${ }^{1}$ Department of Orthopedics, 960th Hospital of PLA, Jinan, Shandong 250031; ${ }^{2}$ Department of Orthopedics, \\ Southwest Hospital, Third Military Medical University, Chongqing 400038, P.R. China
}

Received October 27, 2020; Accepted April 21, 2021

DOI: $10.3892 /$ etm.2021.10600

\begin{abstract}
Tissue-engineered bones (TEB) are a promising strategy for treating large segmental bone defects. However, the application of TEB is greatly limited by technical and logistical issues caused by the viable cells used. The aim of the present study was to devise novel TEB, termed functional TEB (fTEB) using devitalized mesenchymal stem cells (MSCs) with the functional proteins retained. TEB were fabricated by seeding MSCs on demineralized bone matrix (DBM) scaffolds. fTEB were prepared with deep hyperthermia treatment. Total proteins were extracted from fTEB and conditioned media (CM) were prepared. The effects of fTEB-CM on the proliferation, differentiation and migration of host MSCs were assessed. Following lyophilization, the majority of the MSCs were devitalized, but the proteins within the TEB were retained in fTEB. Similar to TEB, fTEB outperformed the DBM in inducing migration, proliferation and osteogenic differentiation in MSCs. The abundance of cytokines in fTEB was also determined. fTEB were shown to be a promising alternative to TEB. Thus, they might serve as off-the-shelf tissue engineering products, meeting the high demands for bone substitutes in the clinical setting.
\end{abstract}

\section{Introduction}

Treatment of large segmental bone defects (LSBD) remains a major challenge in orthopedics. Increasing evidence suggests that tissue-engineered bones (TEB) seeded with osteogenic cells, such as mesenchymal stem cells (MSCs), are promising alternatives to conventional approaches, such as autologous

Correspondence to: Dr Xiuchun Yu, Department of Orthopedics, 960th Hospital of PLA, 25 Shifan Road, Tianqiao, Jinan, Shandong 250031, P.R. China

E-mail: 26766771@qq.com

${ }^{*}$ Contributed equally

Key words: large segmental bone defect, mesenchymal stem cells, tissue-engineered bones, functional tissue-engineered bones, functional proteins or allograft bone grafting for repairing LSBD (1-3). However, TEB are not widely adopted in practice, due to a variety of technical and logistical challenges and the subsequent high cost caused by the need to maintain viability during manufacturing and transportation. In addition, highly-trained technicians are required, inhibiting large-scale production of TEB (4).

The role of MSCs in TEB has previously been challenged; it was reported that MSCs in TEB were almost undetectable 30 days after transplantation and, eventually, almost all newly-formed bones originated from the host (5-7). Another study suggested that MSCs helped create a favorable microenvironment for tissue regeneration instead of contributing to the cellular contents directly (8). MSCs have been shown to secrete a variety of growth factors, chemokines and osteogenic factors, such as C-C motif chemokine ligand 5 (CXCL5), CXCL8 and stromal cell-derived factor 1 (SDF-1) (9). These factors played critical roles in modulating bone repair, including inflammation, angiogenesis, bone formation and remodeling (10). The present study reported a novel strategy, functional TEB (fTEB), to harness the benefit of these factors in TEB by disengaging from viable cells. fTEB were obtained by freeze-drying TEB fabricated with human umbilical cord MSCs (hUC-MSCs) and demineralized bone matrix (DBM) scaffolds. Conditioned media (CM) were prepared from fTEB and their effects on the proliferation, differentiation and migration of host human bone marrow MSCs (hBMSCs) were further assessed.

\section{Materials and methods}

Cell isolation and culture. hUC-MSCs and hBMSCs were isolated, cultured and characterized, as previously described (11). All protocols involving human subjects were approved by the Ethics Committee of the 960th Hospital of PLA (Jinan, China; approval no. JNZY201603), with all subjects providing informed consent. Between January and December 2016, hUC-MSCs were obtained from the umbilical cords of five volunteers (25-35 years old) who underwent a full-term pregnancy (38-40 weeks). hBMSCs were isolated from iliaccrest bone marrow aspirates of five healthy volunteers. Exclusion criteria included malignant tumor or accompanied systemic diseases, such as rheumatoid arthritis, systematic lupus erythematosus and diabetes. Cells derived from these sources were cultured in DMEM/F12 (dilution, 1:1; Cytiva) with 10\% FBS (Gibco; Thermo Fisher 
Scientific, Inc.) and $100 \mathrm{U} / \mathrm{ml}$ of penicillin/streptomycin (Gibco; Thermo Fisher Scientific, Inc.). Cells at passage 3-4 were obtained for use.

Preparation of fTEB. Allogenic DBM scaffolds (Beijing Datsing Bio-Tech Co., Ltd.; Fig. 1A) with a volume porosity of $76 \pm 8.5 \%$ (pore size, $200-800 \mu \mathrm{m}$ ) were cut into blocks (1x1x0.5 cm; $0.417 \mathrm{~g} /$ block) and submerged in DMEM/F12 medium overnight at $4^{\circ} \mathrm{C}$. TEB were fabricated by dropwise instillation of an aliquot $(20 \mu \mathrm{l})$ of single-cell suspension of hUC-MSCs (density, $1 \times 10^{7} / \mathrm{ml}$ ) onto two opposite surfaces of the scaffolds. After $2 \mathrm{~h}$ of incubation at room temperature, media were added and replaced every 2 days. The seeding efficiency was assayed at $10 \mathrm{~h}$ by calculating the ratio of the number of cells retained in the scaffold to the number of cells seeded. The number of retained cells in the scaffold was defined as the difference between the cells seeded and the residual cells in the medium. hUC-MSCs retained on the scaffolds were visualized on days 5 and 10, by scanning electron microscopy (SEM; Hitachi, Ltd.). In addition, the cytoskeletons and nuclei of hUC-MSCs were stained with $25 \mathrm{mg} / \mathrm{ml}$ rhodamine-labelled phalloidin (Biotium, Inc.) for $1 \mathrm{~h}$ and $10 \mathrm{mg} / \mathrm{ml}$ DAPI (Merck KGaA) for $15 \mathrm{~min}$ at room temperature, respectively. Samples were then subjected to confocal laser scanning microscopy (Leica Microsystems $\mathrm{GmbH}$ ). To fabricate fTEB, TEB were washed three times with PBS, immersed in PBS for $2 \mathrm{~h}$ and frozen at $-80^{\circ} \mathrm{C}$ for $2 \mathrm{~h}$. Samples were then freeze-dried using a deep-hypothermic lyophilizer (Modulyo ${ }^{\circledR}$; Thermo Fisher Scientific, Inc.) for $4 \mathrm{~h}$ and stored at $-80^{\circ} \mathrm{C}$.

Preparation of total protein content and CM. In total, $1 \mathrm{~cm}^{3}$ fTEB were used for protein extraction. Samples were homogenized in liquid nitrogen. Total proteins were extracted using RIPA Buffer (Beyotime Institute of Biotechnology) and protein concentrations were determined using a BCA Protein Assay kit (Beyotime Institute of Biotechnology). CM were prepared by dissolving total protein extracted from $1 \mathrm{~cm}^{3}$ fTEB in $10 \mathrm{ml}$ DMEM/F12 complete media. CM of TEB and DBM were also prepared using the same method.

Quantitative human cytokine array. A customized Quantibody ${ }^{\circledR}$ human cytokine array (QAH-CUSTOM; RayBiotech,Inc.) was performed for 49 cytokines. The cytokines of interest are presented in Table SI. The fTEB-CM (100 $\mu \mathrm{l})$ was assayed according to the manufacturer's instructions. Fluorescent signals were detected using a GenePix ${ }^{\mathrm{TM}} 4000 \mathrm{~B}$ laser scanner (Molecular Devices, LLC). Data were extracted and analyzed using RayBio QAM-TH17-1software (RayBiotech, Inc.).

Migration assay. Migration assays were performed in Transwell inserts (8- $\mu \mathrm{m}$ pores; Corning, Inc.). Briefly, $700 \mu \mathrm{l}$ fTEB-CM was added to the lower chamber. A total of $2 \times 10^{4} \mathrm{hBMSCs}$ in $200 \mu 1$ serum-free medium (Cytiva) were seeded in the upper chamber and allowed to migrate for $48 \mathrm{~h}$ at $37^{\circ} \mathrm{C}$. Next, cells in the upper chamber (non-migrating cells) were removed using a cotton wool swab. Migrated cells in the lower chamber were washed with PBS, fixed with $4 \%$ paraformaldehyde (Wuhan Boster Biological Technology, Ltd.), stained with DAPI for $15 \mathrm{~min}$ at room temperature and observed under a fluorescent microscope (Olympus Corporation). The number of migrated cells was counted from 10 random high-power fields (magnification x100) and the mean taken. TEB-CM, DBM-CM and DMEM/F12 containing recombinant human SDF-1 $(20 \mathrm{ng} / \mathrm{ml})$ served as controls. Migration assays were performed on five batches of hBMSCs from different donors.

Proliferation assay. The proliferation of hBMSCs was evaluated by Cell Counting Kit-8 assay (CCK-8; Dojindo Molecular Technologies, Inc.). hBMSCs were harvested and resuspended with different CMs (fTEB-CM, TEB-CM and DBM-CM) and basic culture media (BCM) at a density of $1 \times 10^{5}$ cells $/ \mathrm{ml}$. A total of $0.1-\mathrm{ml}$ aliquots of hBMSC suspension was seeded in 24-well plates. At each time point (0, 12, 24, 48, 72 and $96 \mathrm{~h})$, CCK-8 reagent was added and the absorbance was measured at $450 \mathrm{~nm}$ using a microplate reader. The survival of hBMSCs was represented as the optical density (OD) value of each well. The growth curve of cells was plotted and population doubling time (PDT) was calculated using the following formula: $\mathrm{PDT}=\mathrm{h}$ of exponential phase $/[(\log \mathrm{N} 2-\log \mathrm{N} 1) / \log 2]$, where $\mathrm{N} 1$ is the number of cells at the beginning of the exponential growth phase and N2 is the number of cells at the end of the exponential growth phase.

Evaluation of osteogenic differentiation. Briefly, hBMSCs were seeded into 6-well plates (density, $5 \times 10^{3} / \mathrm{cm}^{2}$ ). Osteogenic differentiation was induced by incubation with different media, including fTEB-CM, TEB-CM and DBM-CM, and osteogenic medium (OM; Cytiva). After 14 days, the osteogenic differentiation potential was verified by Alizarin Red staining (Cytiva) according to the manufacturer's instructions. Osteogenic genes and proteins were analyzed by reverse transcription (RT) semi-quantitative PCR and western blot (WB) analysis, respectively.

RT semi-quantitative PCR. Cells were obtained and total RNA was extracted using Qiagen RNeasy Mini kits (Qiagen AB). cDNA was prepared from $1 \mu \mathrm{g}$ total RNA using cDNA synthesis kits (Promega Corporation) according to the manufacturer's instructions. RT semi-quantitative PCR was performed using SYBR ExScript RT-PCR kits (PerfectRealTime; Takara Bio, Inc.) 18 s ribosomal RNA served as the internal control. The primers used are presented in Table I.

WB analysis. Cells were collected, lysed with SDS lysis buffer [100 mM Tris (Ph 8.0), 10\% glycerol and 1\% SDS; Beyotime Institute of Biotechnology] on ice and then centrifuged at $10,000 \mathrm{x} \mathrm{g}$ for $1 \mathrm{~min}$ at $4^{\circ} \mathrm{C}$. The supernatant was obtained, centrifuged at $10,000 \mathrm{x} \mathrm{g}$ for $1 \mathrm{~min}$ at $4^{\circ} \mathrm{C}$ and stored at $-20^{\circ} \mathrm{C}$. Each SDS-PAGE (Beyotime Institute of Biotechnology) lane was loaded with $20 \mu \mathrm{g}$ protein. The gel electrophoresis conditions were polyacrylamide $(10 \%, \mathrm{v} / \mathrm{v}), 80 \mathrm{~V}$ and $90 \mathrm{~min}$. The gel was then transferred onto PVDF membranes $(250 \mathrm{~mA}$ for 60 min; EMD Millipore). After blocking with 5\% skimmed milk for $1 \mathrm{~h}$ at room temperature, the membranes were incubated overnight at $4^{\circ} \mathrm{C}$ with the primary antibodies, including anti-RUNX family transcription factor 2 (RUNX2; cat. no. ab236639; 1:1,000; Abcam), anti-alkaline phosphatase (ALP; cat. no. ab154100; 1:2,000; Abcam), anti-amyloid fibrils (OC; cat. no. ab133612; 1:4,000; Abcam) and $\beta$-actin 
Table I. Reverse transcription semi-quantitative PCR primers.

Primer $\left(5^{\prime}-3^{\prime}\right)$

\begin{tabular}{lll}
\cline { 2 - 3 } Gene & \multicolumn{1}{c}{ Forward } & \multicolumn{1}{c}{ Reverse } \\
\hline ALP & CCCACAATGTGGACTACCT & GAAGCCTTTGGGGTTCTTC \\
RUNX2 & CGGAGTGGACGAGGCAAGAG & TGAGGAATGCGCCCTAAATC \\
OC & CACTCCTCGCCCTATTGGCC & CCTCCTGCTTGGACACAAAG \\
$18 \mathrm{~s}$ rRNA & GTAACCCGTTGAACCCCATT & CCATCCAATCGGTAGTAGCG
\end{tabular}

ALP, alkaline phosphatase; RUNX2, RUNX family transcription factor 2; OC, osteocalcin; rRNA, ribosomal RNA.

(cat. no. ab8227; 1:4,000; Abcam). After washing with TBST (Beyotime Institute of Biotechnology) under oscillation for 15 min for 3 times, the peroxidase-conjugated secondary antibody (cat. no. AP132P; 1:4,000; SouthernBiotech) was added and incubated at room temperature for $1 \mathrm{~h}$. The membrane was washed with TBST for 15 min for 3 times and signals were then detected by ECL (Kirkegaard \& Perry Laboratories Inc.). When the bands were developed, images were captured and ImageJ V1.8.0 (NIH) used for analysis.

Statistical analysis. All values are expressed as means \pm SD. Statistical analysis was performed using SPSS 13.0 software (SPSS, Inc.). The results of migration, proliferation and differentiation assays among groups were compared using one-way ANOVA with the Bonferroni correction as a post hoc test, where $\mathrm{P}<0.0083$ was considered to indicate a statistically significant difference. The results of cytokine antibody arrays were analyzed by one-way ANOVA with Tukey's post hoc test. $\mathrm{P}<0.05$ was considered to indicate a statistically significant difference.

\section{Results}

hUC-MSCs in TEB. The mean efficiency of cell seeding was $82.4 \pm 3.3 \%(n=10)$. On day 3 after cell seeding, hUC-MSCs spread and attached well on the pore surfaces of the scaffolds, with dense extracellular matrix deposition (Fig. 1B). With the proliferation of hUC-MSCs and production of the extracellular matrix, cells were stacked and superimposed over each other on day 10 (Fig. 1C). Confocal microscopy further confirmed that hUC-MSCs were tightly attached to the scaffolds after 3 (Fig. 1E) and 10 (Fig. 1F) days. It was revealed by SEM that although hUC-MSCs were devitalized by freeze-drying, the remnant cellular contents were retained on the pore surfaces of fTEB (Fig. 1D).

The majority of proteins are retained following freeze-drying. To assess the effect of lyophilization on the protein loss of TEB, the protein content was monitored following cell seeding. The protein contents of TEB and the derived fTEB increased over time and peaked on day 12. Thus, fTEB prepared 12 days after cell seeding were harvested for subsequent use. At any given time-point, the protein content of TEB was significantly higher than that of fTEB $(\mathrm{P}<0.05)$. However, the mean retention rate from 10 samples, which was defined as the ratio between protein contents of fTEB and TEB harvested following a 10-day culture was 64.0\% (60.4-66.7\%; Fig. $1 \mathrm{G}$ and $\mathrm{H})$.

Certain cytokines in fTEB might be responsible for its biological function. To identify the proteins accounting for the functions of fTEB, a customized Quantibody ${ }^{\circledR}$ human cytokine array (QAH-CUSTOM; RayBiotech, Inc.) was performed. The levels of the majority of proteins of interest were significantly higher in fTEB, as compared with DBM (Fig. 1H). Only those with concentrations of $>10 \mathrm{ng} / \mathrm{ml}$ were selected and presented in Fig. 1H. The maximal concentration occurred in basic fibroblast growth factor (bFGF) with a mean of $525.42 \mathrm{ng} / \mathrm{ml}$ in fTEB $(n=4)$.

fTEB shows a marked capability in inducing hBMSC migration. To investigate the hBMSC-recruiting capacity of fTEB, migration assays were performed. As demonstrated in Fig. 2, the chemotactic activity of fTEB was significantly higher than that of TEB, SDF-1 and DBM $(\mathrm{P}<0.0083)$.

fTEB exerts positive effects on the proliferation of $h B M S C s$. To evaluate the biological role of fTEB in the proliferation of hBMSCs, cell growth kinetics were evaluated. CCK-8 assays demonstrated that, after $24 \mathrm{~h}$ of culture, fTEB-CM and TEB-CM promoted the proliferation of hBMSCs, as evidenced by the increased OD values when compared to the BCM and DBM-CM (Fig. 3). The proliferation-promoting capacity of fTEB-CM was not significantly different to TEB-CM $(\mathrm{P}>0.05)$ at all time points but $96 \mathrm{~h}$. According to the growth curve, hBMSCs cultured with fTEB-CM and TEB-CM displayed a similar growth pattern, with a lag stage and an extensive log phase lasting $\sim 2$ and $\sim 4$ days, respectively. The subsequent platform phase occurred at day 8. Moreover, the growth rates of hBMSCs cultured with BCM and DBM-CM were significantly lower than those of the fTEB-CM and TEB-CM groups $(\mathrm{P}<0.05)$. In addition, no apparent difference in the mean PDT was observed between fTEB-CM $(28.24 \pm 6.51 \mathrm{~h})$ and TEB-CM (26.68 $\pm 4.32 \mathrm{~h} ; \mathrm{P}>0.05)$. However, both were significantly lower than BCM $(36.69 \pm 8.92 \mathrm{~h})$ and DBM-CM (123.52 $\pm 13.50 \mathrm{~h} ; \mathrm{P}<0.05)$.

fTEB exhibit comparable osteogenic capacity to TEB. Microscopic examination of Alizarin Red staining showed clear crimson deposits indicative of mineralization following incubation with fTEB-CM, TEB-CM and OM, but not with 
A

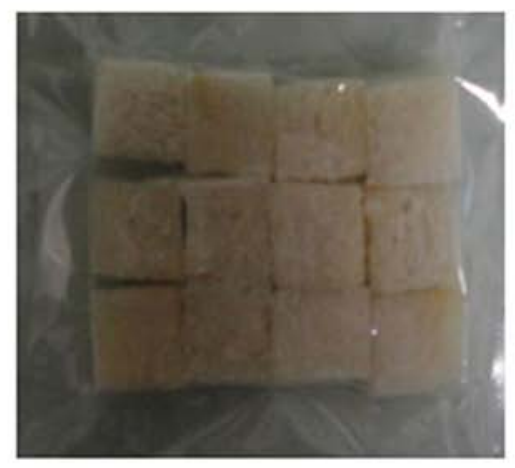

C

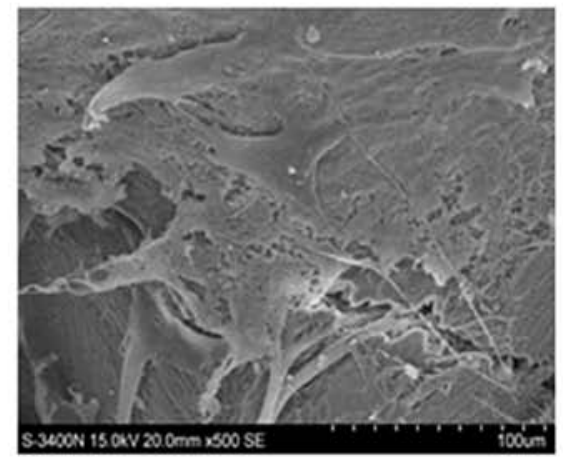

E

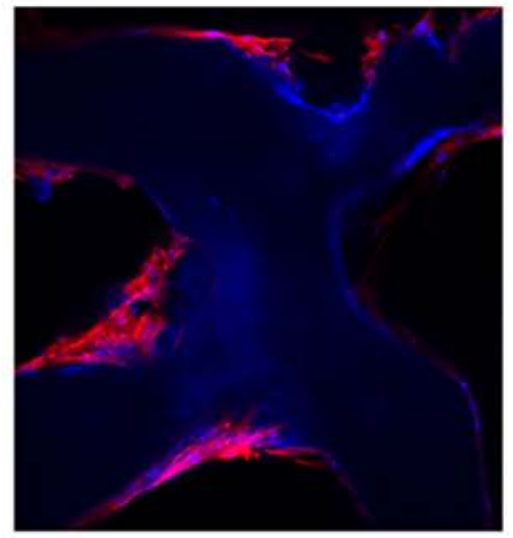

G

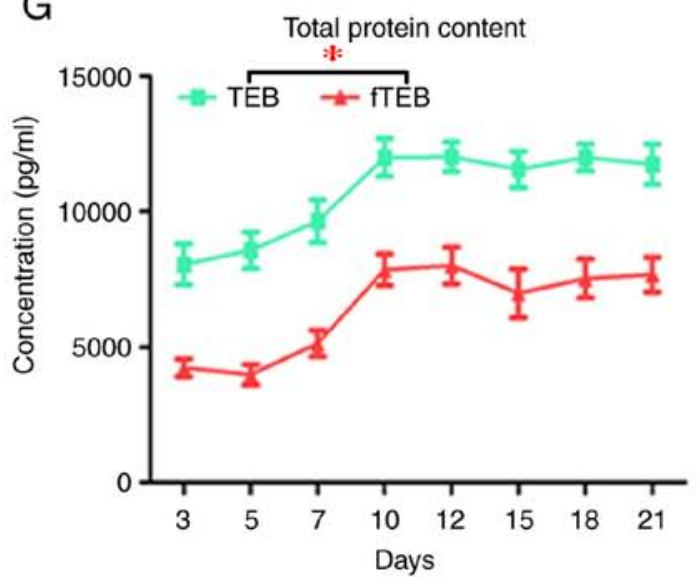

B

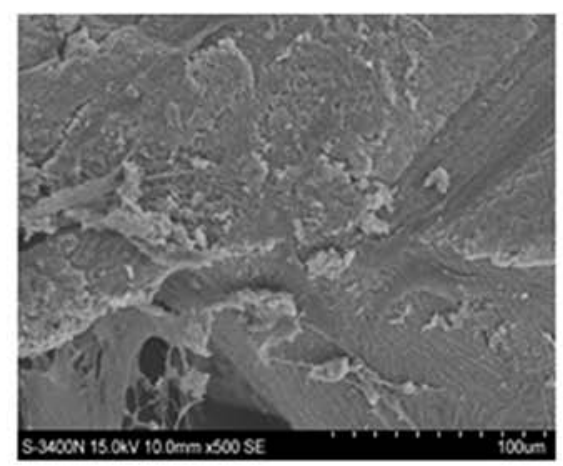

D

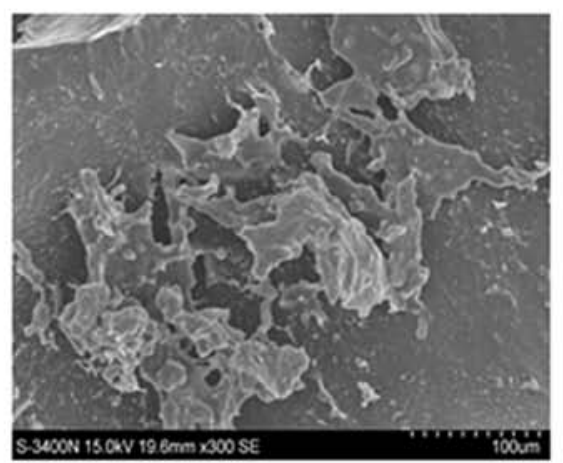

F

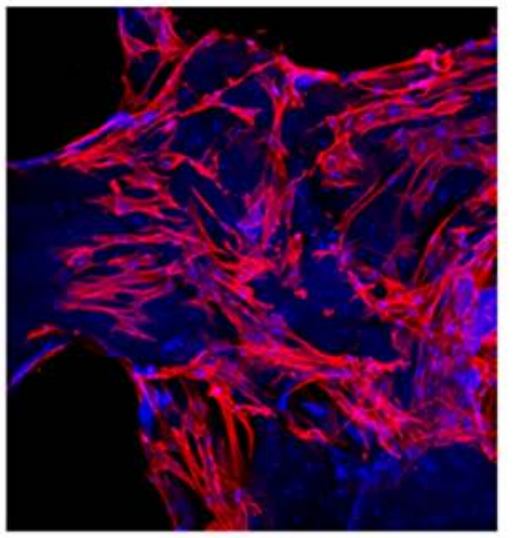

H

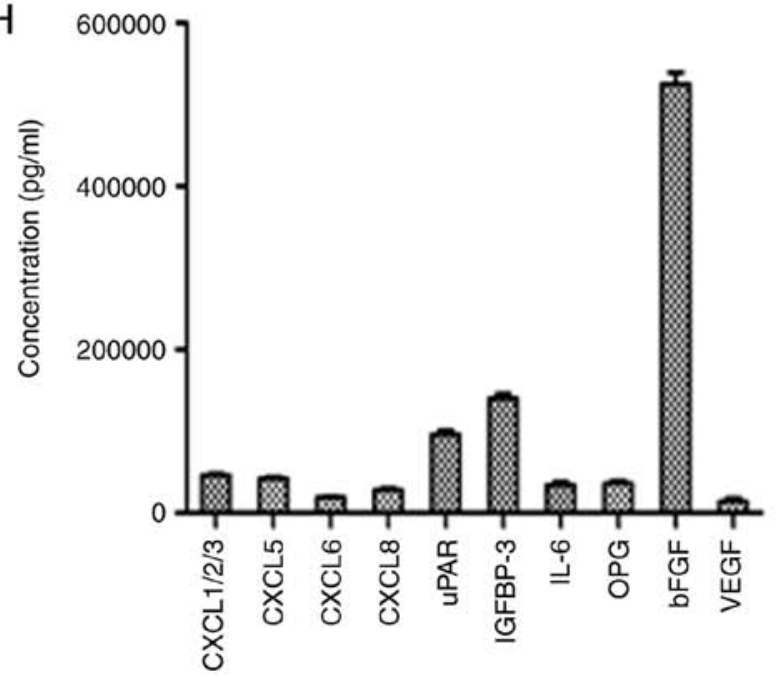

Figure 1. Adhesion and growth of cells on DBM scaffolds. (A) Gross image of DBM scaffolds. (B) A total of 3 days after seeding, cells adhered to the inner surfaces of the scaffolds with dense extracellular matrix deposition (magnification x100). (C) After 10 days of culture, cells were tightly interwoven and stacked on top of one another (magnification x100). (D) In fTEB obtained on day 10, the exposed cellular contents had been retained at the pore surfaces. (E and F) Confocal microscopy further confirmed that cells were tightly attached to the scaffolds after 3 and 10 days (magnification x100). (G) Total protein contents of TEB and fTEB over 10 days. ${ }^{*} \mathrm{P}<0.001$ (H) The top 10 cytokines identified in fTEB ( $\left.>10 \mathrm{ng} / \mathrm{ml}\right)$. DBM, demineralized bone matrix; TEB, tissue-engineered bones; fTEB, functional TEB; CXCL, C-C motif chemokine ligand; uPAR, urokinase type plasminogen activator receptor; IGFBP-3, insulin-like growth factor binding protein 3; IL-6, interleukin 6; OPG, osteoprotegerin; bFGF, basic fibroblast growth factor. 


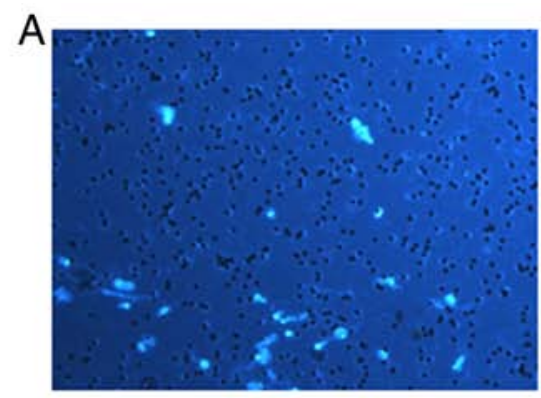

TEB

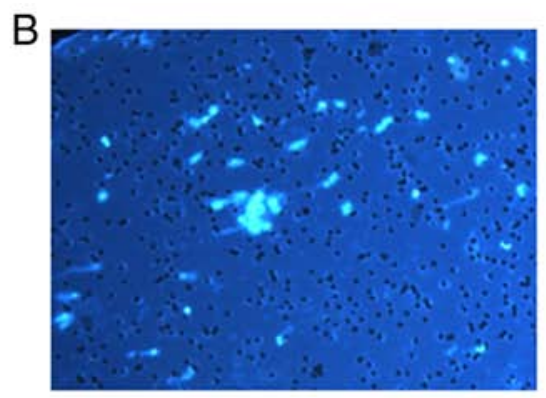

fTEB

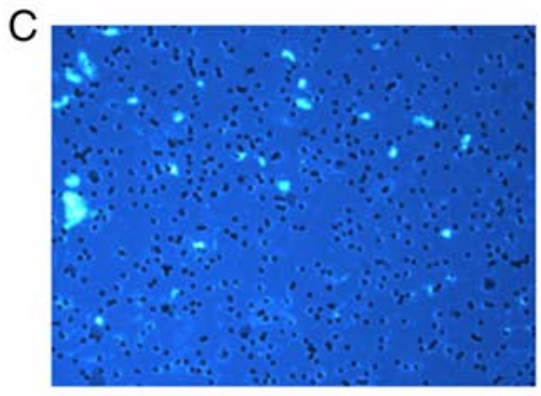

SDF-1

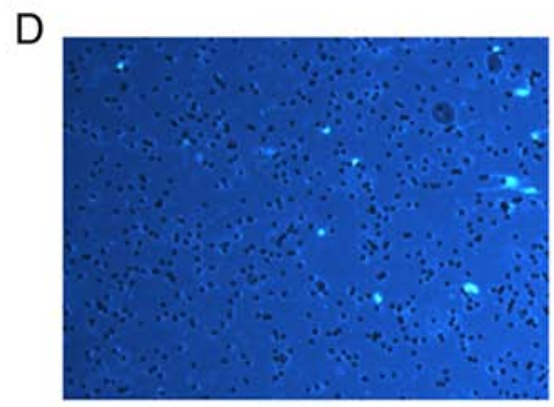

$\mathrm{E}$

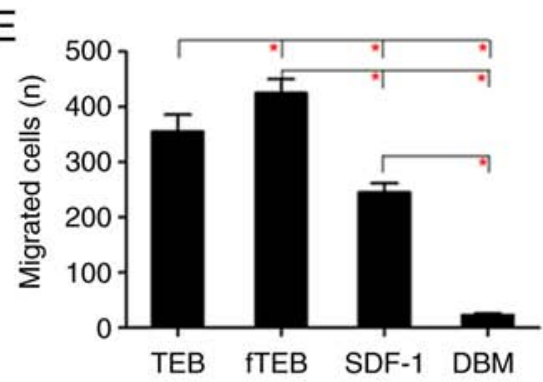

DBM

Figure 2. Migration of human bone marrow mesenchymal stem cells with different conditioned media. (A-D) Images of in vitro migration assay using the Transwell culture system. (magnification x40). (E) Quantification of Transwell results. The bars represent means $\pm \mathrm{SD}(\mathrm{n}=10)$ ) ${ }^{*} \mathrm{P}<0.0083$. TEB, tissue-engineered bones; fTEB, functional TEB; SDF-1, stromal cell-derived factor 1; DBM, demineralized bone matrix.
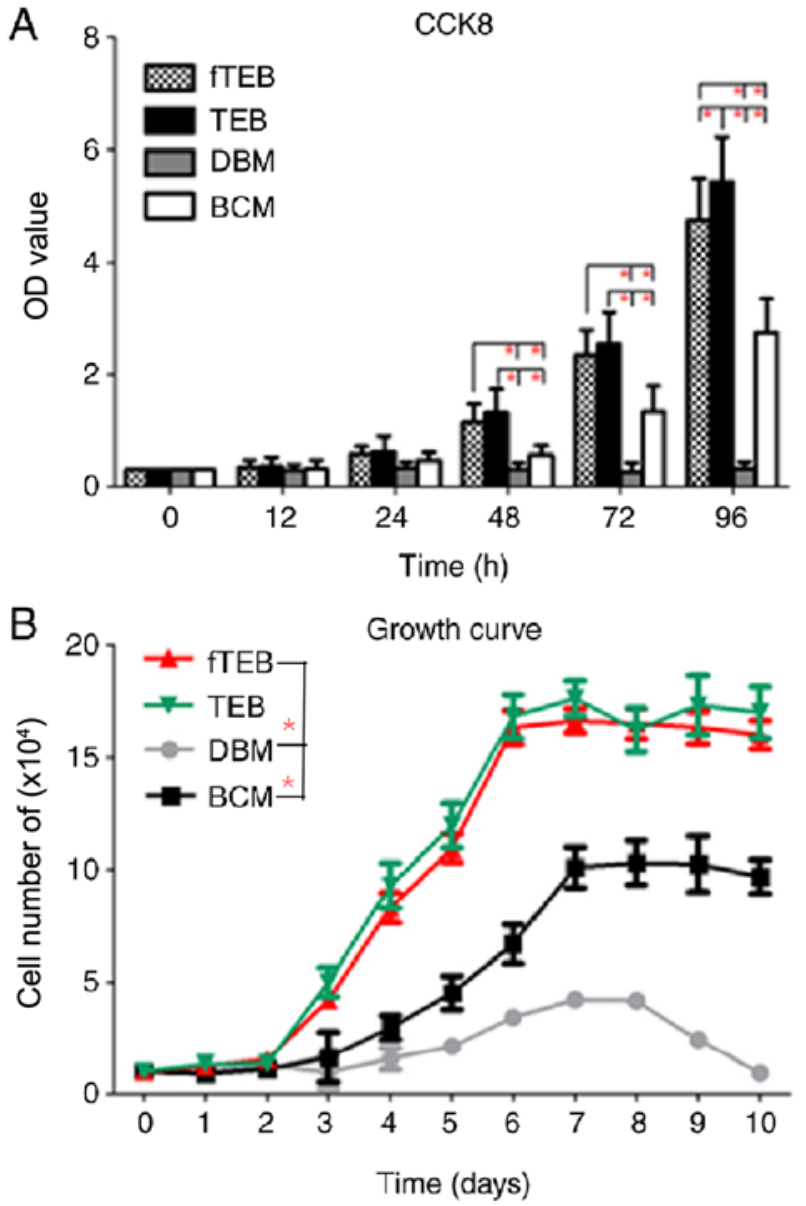

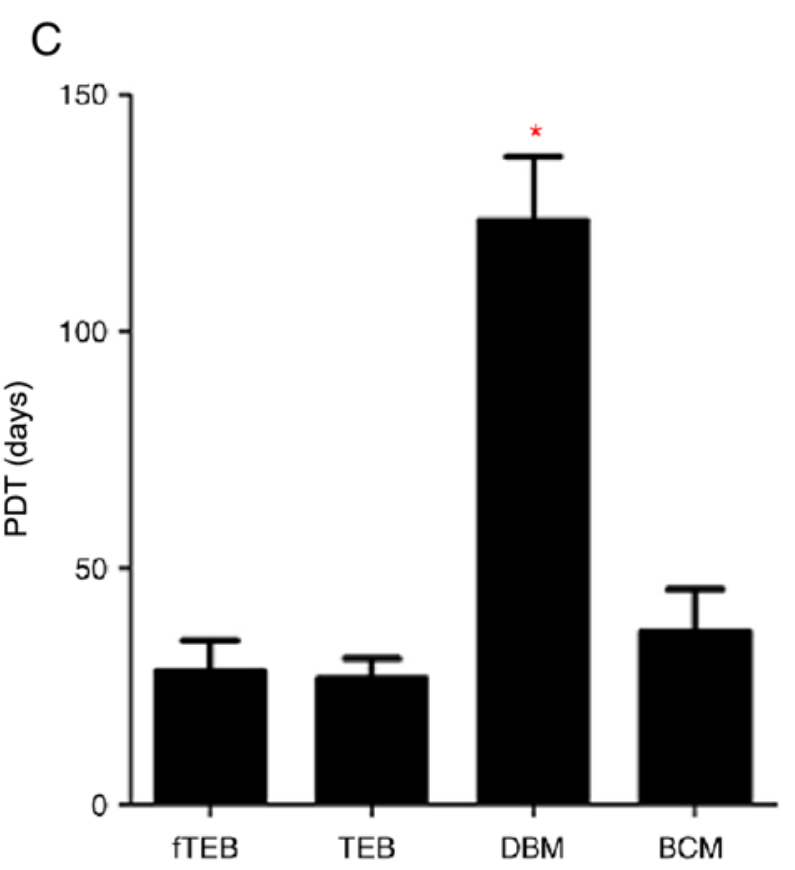

Figure 3. Proliferation assays. (A) fTEB-CM showed comparable pro-proliferation effects on hBMSCs in TEB-CM. (B) Growth curves of cells treated with fTEB, TEB-CM, DBM and BCM. (C) Mean PDT of cells treated with fTEB-CM, TEB-CM, DBM or BCM. "P<0.0083. TEB, tissue-engineered bones; fTEB, functional TEB; CM, conditioned media; BCM, basic culture media; PDT, population doubling time; hBMSCs, human bone marrow mesenchymal stem cells; DBM, demineralized bone matrix. 


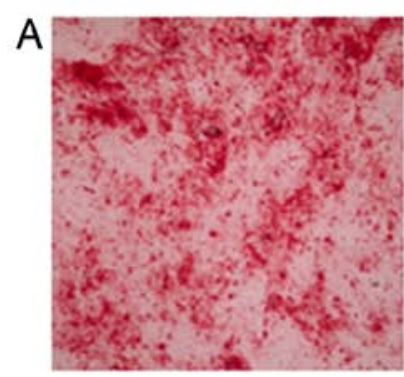

B
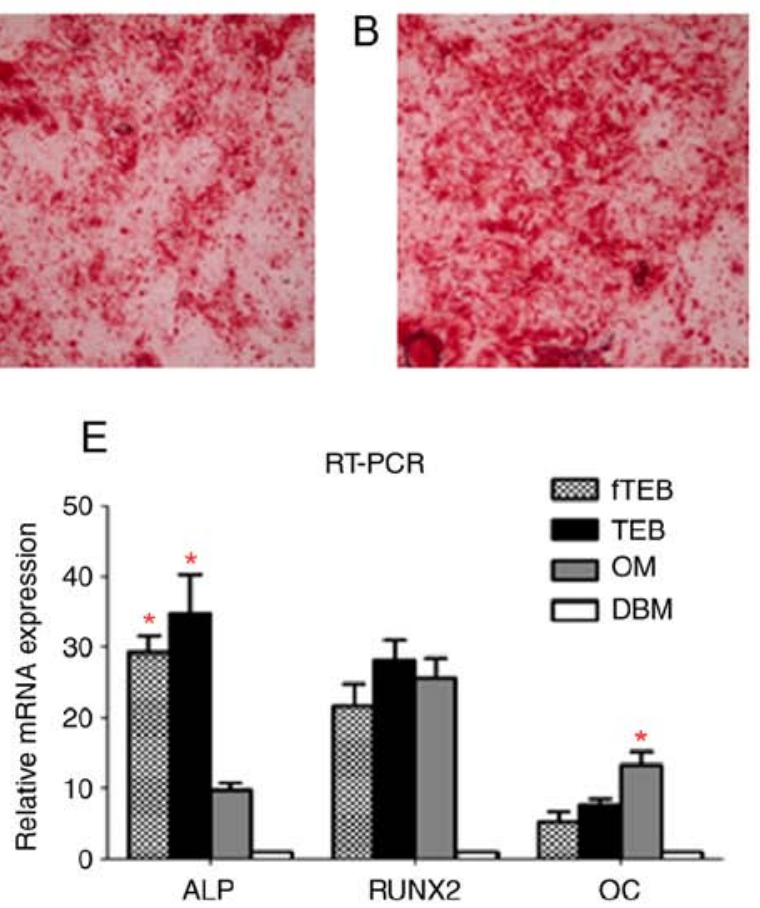
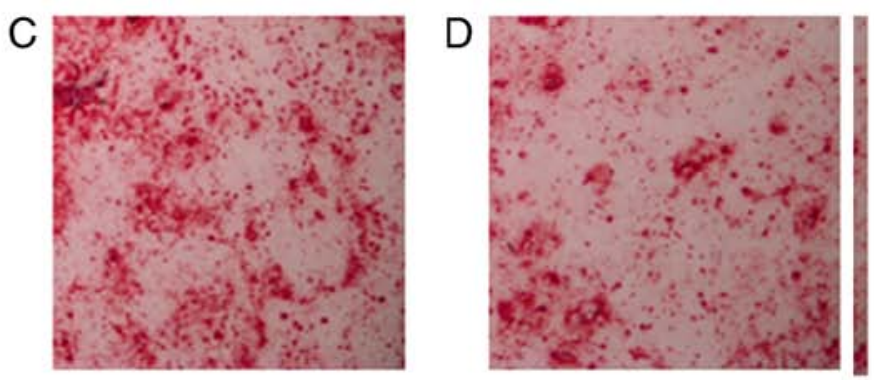

$\mathrm{F}$

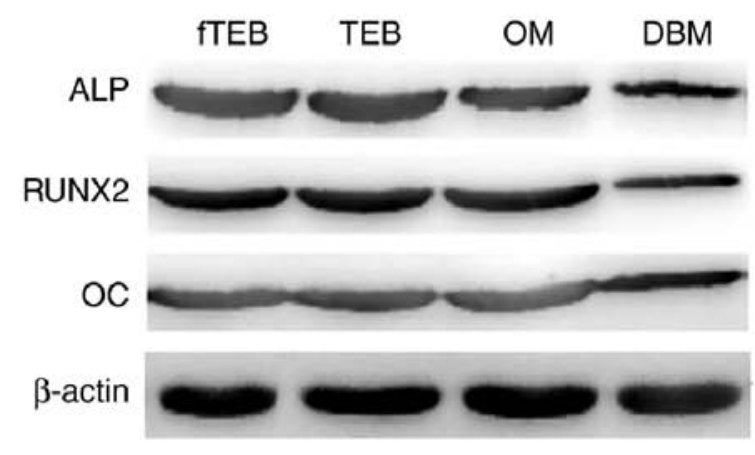

Figure 4. Comparison of osteogenic differentiation between hBMSCs treated with different CM. (A-D) Microscopic examination of Alizarin Red staining (magnification x40). (E) ALP, RUNX2 and OC mRNA expression levels in cells treated with different CM (n=5). ALP mRNA expression levels were similar in cells treated with fTEB-CM and TEB-CM, but significantly higher than those in OM-treated cells $\left({ }^{*} \mathrm{P}<0.05\right)$. OM induced a higher OC mRNA expression level than fTEB-CM and TEB-CM ( $\mathrm{P}<0.05)$. In addition, the difference between RUNX2 mRNA expression levels in the fTEB-CM, TEB-CM and OM groups were not significantly different $(\mathrm{P}>0.05)$. (F) Protein expression of ALP, RUNX2 and OC ( $\mathrm{n}=5)$. Bars represent the mean \pm SD. hBMSCs, human bone marrow mesenchymal stem cells; ALP, alkaline phosphatase; RUNX2, RUNX family transcription factor 2; OC, anti-amyloid fibrils; OM, osteogenic medium; TEB, tissue-engineered bones; fTEB, functional TEB; CM, conditioned media.

DBM-CM (Fig. 4A-D). The expression levels of osteocyte markers were semi-quantitatively evaluated by RT-PCR (Fig. 4E). ALP mRNA expression levels were similar in cells treated with fTEB-CM and TEB-CM, but significantly higher than those in $\mathrm{OM}$-treated cells $(\mathrm{P}<0.05)$. OM induced a higher OC mRNA expression level than fTEB-CM and TEB-CM $(\mathrm{P}<0.05)$. In addition, the difference between RUNX2 mRNA expression levels in the fTEB-CM, TEB-CM and OM groups were not significantly different $(\mathrm{P}>0.05)$. A similar expression pattern was also observed at the protein level (Fig. 4F). In combination, these results demonstrated that fTEB were not significantly different to TEB with regard to inducing osteogenic differentiation of hBMSCs.

\section{Discussion}

The application of TEB in the clinical setting has been limited by several crucial hurdles, such as the requirement of long-term in vitro cell culture, high cost, safety concerns and logistical challenges (8). In the present study, a novel TEB device, fTEB, which is based on the concept that implanted MSCs aid bone repair via paracrine modes, rather than direct osteogenic differentiation, was introduced (12-14). Briefly, fTEB were prepared by freeze-drying TEB with allogenic hUC-MSCs. Using a deep-hypothermic lyophilizer, cells were devitalized while most protein content was retained. It is important to note that if the major contributing components were retained the aforementioned obstacles in the application of TEB could be greatly mitigated (15).
The role of exogenous MSCs within TEB in bone repair remains unclear. Previous studies indicate that, upon implantation, exogenous MSCs recruit host stem cells and modulate their biological behaviors viacytokines (5-7). Moreover, it is widely accepted that the mobilization and homing of endogenous progenitor cells (such as MSCs) to the injury site is a prerequisite for tissue regeneration (16). Our previous study identified a series of chemokines associated with the recruitment of host MSCs mediated by donor MSCs (9). In the present study, the MSC-recruiting capacity of fTEB was similar to that of TEB, suggesting that most of the functional chemoattractants were retained following freeze-drying. It was found that growth-regulated oncogene (GRO; $46.08 \mathrm{ng} / \mathrm{ml}$ ), CXCL5 (41.89 ng/ml), CXCL6 $(18.89 \mathrm{ng} / \mathrm{ml})$ and CXCL8 $(27.89 \mathrm{ng} / \mathrm{ml})$ might contribute to the MSC-recruiting capacity of fTEB. The GRO subgroup belongs to the CXCL8 cytokine family and consists of three members, CXCL1/GRO- $\alpha$, CXCL2/GRO- $\beta$ and CXCL3/GRO- $\gamma$ (17). By binding CXCR2, a cell-surface cognate receptor constitutively expressed by hBMSCs, these chemokines play crucial roles in cell mobilization and homing $(18,19)$. Notably, CXCR2 is not only a co-receptor of the CXCL8 family of cytokines, but also binds to CXCL5 and CXCL6 (20). Downstream signaling pathways, including the PI3K-Akt and mTOR signaling pathways, are activated by CXCR2, thus regulating cell migration (18). In addition, it is worth noting that the recruiting capacity of fTEB-CM was more potent than that of a well-defined MSC chemoattractant, SDF-1, which was demonstrated in the present study. 
During bone defect repair, implanted TEB generate trophic factors to modulate the surrounding microenvironment and promote tissue regeneration (21). In the present study, it was found that fTEB-CM exerted comparable effects on the proliferation and osteogenic differentiation of hBMSCs to those of TEB-CM. This was further supported by the cytokine array, as the concentrations of certain growth factors associated with cell proliferation and differentiation were high in fTEB-CM. According to the literature, bFGF appears to be the main facilitator of cell proliferation (21). To further support this notion, the proliferation-promoting effect of bFGF on MSCs had been well-recognized in previous studies (22-25) and, in the present study, a high concentration of bFGF was observed in fTEB-CM (higher than the concentration used in previous studies) (22-25). Conversely, identifying a predominant factor accounting for the enhanced osteogenic differentiation was challenging. Insulin-like growth factor-binding protein 3, interleukin 6 and vascular endothelial growth factor (VEGF) have all been shown to have the ability to potentiate osteogenic differentiation (26-28). In addition, bFGF has been reported to promote osteogenic differentiation in the presence of bone morphogenetic protein 2 (BMP-2) (23), BMP-2 and VEGF (29) or BMP-4/7 (30), but to inhibit osteogenic differentiation on its own or with EGF (31). These results further complicated the compound effects induced by cytokine combinations. The regulation of osteogenic repair involves multiple factors, including cells and various cytokines. Currently, the integration of factors into DBM has been limited to no more than three factors. However, there are 49 known factors in fTEB, indicating its comprehensive and synergetic effects on osteogenesis.

It must be noted that the present study had certain limitations. The immunogenicity of fTEB was not evaluated. Furthermore, freeze-drying damaged the cell membrane, which may have exposed immunogenic intracellular proteins to the immune system. Additionally, the in vivo safety and efficacy of fTEB were not investigated in the present study. Obtaining an understanding of this issue is critical to the further development of fTEB and relevant animal studies are ongoing. Finally, this was a preliminary study, the next step will be to verify the key cytokines modulating the biological behaviors of MSCs and elucidate the correlation between them.

Thus, the present study introduced a prototype of an fTEB device, which could easily be fabricated by freeze-drying matured TEB with a widely available deep-hypothermic lyophilizer. These results collectively supported the hypothesis that compared to TEB, fTEB may be more economical and easier to manufacture. However, further validation of the efficacy and safety of fTEB is warranted in future studies.

\section{Acknowledgements}

Not applicable.

\section{Funding}

The present study was supported by the China Postdoctoral Science Foundation (grant. no. 2015M572713), the Natural Science Foundation of China (grant. no. 81971762) and the President Funding of the 960th Hospital of PLA (grant. no. 2013ZD02).

\section{Availability of data and materials}

The datasets used and/or analyzed during the current study are available from the corresponding author on reasonable request.

\section{Authors' contributions}

ZC and JX contributed equally to this work. ZC and JX made substantial contributions to acquisition, analysis and interpretation of data. XY was responsible for the conception and design of the study and the drafting and writing of this manuscript. All authors had read and approved the final manuscript. All authors confirm the authenticity of all the raw data.

\section{Ethics approval and consent to participate}

All protocols involving human subjects were approved by the Ethics Committee of the 960th Hospital of PLA (approval. no. JNZY201603). All patients were asked to sign an informed consent statement for publication.

\section{Patient consent for publication}

All patients agreed to publish the data and associated images.

\section{Competing interests}

The authors declare that they have no competing interests.

\section{References}

1. Quarto R, Mastrogiacomo M, Cancedda R, Kutepov SM, Mukhachev V, Lavroukov A, Kon E and Marcacci M: Repair of large bone defects with the use of autologous bone marrow stromal cells. New Engl J Med 344: 385-386, 2001.

2. Xu JZ, Qin H, Wang XQ, Zhou Q, Luo F, Hou TY and He QY: Repair of large segmental bone defects using bone marrow stromal cells with demineralized bone matrix. Orthop Surg 1: 34-41, 2009.

3. Kitoh H, Kitakoji T, Tsuchiya H, Katoh $\mathrm{M}$ and Ishiguro $\mathrm{N}$ : Distraction osteogenesis of the lower extremity in patients with achondroplasia/hypochondroplasia treated with transplantation of culture-expanded bone marrow cells and platelet-rich plasma. J Pediatr Orthop 27: 629-634, 2007.

4. Xing J, Lu Y, Cui Y, Zhu X, Luo F, Xie Z, Wu X, Deng M, Xu J and Hou T: A standardized and quality-controllable protocol of constructing individual tissue-engineered grafts applicable to treating large bone defects. Tissue Eng Part C Methods 25: $137-147,2019$.

5. Tasso R, Augello A, Boccardo S, Salvi S, Carida M, Postiglione F, Fais F, Truini M, Cancedda R and Pennesi G: Recruitment of a host's osteoprogenitor cells using exogenous mesenchymal stem cells seeded on porous ceramic. Tissue Eng Part A 15: 2203-2212, 2009.

6. Tortelli F, Tasso R, Loiacono F and Cancedda R: The development of tissue-engineered bone of different origin through endochondral and intramembranous ossification following the implantation of mesenchymal stem cells and osteoblasts in a murine model. Biomaterials 31: 242-249, 2010.

7. Becquart P, Cambon-Binder A, Monfoulet LE, Bourguignon M, Vandamme K, Bensidhoum M, Petite H and Logeart-Avramoglou D: Ischemia is the prime but not the only cause of human multipotent stromal cell death in tissue-engineered constructs in vivo. Tissue Eng Part A 18: 2084-2094, 2012. 
8. Amini AR, Laurencin CT and Nukavarapu SP: Bone tissue engineering: Recent advances and challenges. Crit Rev Biomed Eng 40: 363-408, 2012.

9. Xing J, Hou T, Jin H, Luo F, Change Z, Li Z, Xie Z and Xu J: Inflammatory microenvironment changes the secretory profile of mesenchymal stem cells to recruit mesenchymal stem cells. Cell Physiol Biochem 33: 905-919, 2014.

10. Habib HS, Halawa TF and Atta HM: Therapeutic applications of mesenchymal stroma cells in pediatric diseases: Current aspects and future perspectives. Med Sci Monit 17: RA233-RA239, 2011

11. Hou T, Xu J, Wu X, Xie Z, Luo F, Zhang Z and Zeng L: Umbilica cord Wharton's Jelly: A new potential cell source of mesenchymal stromal cells for bone tissue engineering. Tissue Eng Part A 15: 2325-2334, 2009.

12. Horwitz EM, Prockop DJ, Fitzpatrick LA, Koo WW, Gordon PL, Neel M, Sussman M, Orchard P, Marx JC, Pyeritz RE and Brenner MK: Transplantability and therapeutic effects of bone marrow-derived mesenchymal cells in children with osteogenesis imperfecta. Nat Med 5: 309-313, 1999.

13. Horwitz EM, Gordon PL, Koo WK, Marx JC, Neel MD, McNall RY, Muul L and Hofmann T: Isolated allogeneic bone marrow-derived mesenchymal cells engraft and stimulate growth in children with osteogenesis imperfecta: Implications for cell therapy of bone. Proc Natl Acad Sci USA 99: 8932-8937, 2002.

14. Gnecchi M, Zhang Z, Ni A and Dzau VJ: Paracrine mechanisms in adult stem cell signaling and therapy. Circ Res 103: 1204-1219, 2008

15. Li W, Liu Y, Zhang P, Tang Y, Zhou M, Jiang W, Zhang X, Wu G and Zhou Y: Tissue-engineered bone immobilized with human adipose stem cells-derived exosomes promotes bone regeneration. ACS Appl Mater Interfaces 10: 5240-5254, 2019.

16. Chen FM, Wu LA, Zhang M, Zhang R and Sun HH: Homing of endogenous stem/progenitor cells for in situ tissue regeneration: Promises, strategies, and translational perspectives. Biomaterials 32: 3189-3209, 2011.

17. Chen HW, Chen HY, Wang LT, Wang FH, Fang LW, Lai HY, Chen HH, Lu J, Hung MS, Cheng Y, et al: Mesenchymal stem cells tune the development of monocyte-derived dendritic cells toward a myeloid-derived suppressive phenotype through growth-regulated oncogene chemokines. J Immunol 190: 5065-5077, 2013.

18. Ringe J, Strassburg S, Neumann K, Endres M, Notter M, Burmester GR, Kaps C and Sittinger M: Towards in situ tissue repair: Human mesenchymal stem cells express chemokine receptors CXCR1, CXCR2 and CCR2, and migrate upon stimulation with CXCL8 but not CCL2. J Cell Biochem 101: 135-146, 2007.

19. Keeley EC, Mehrad B and Strieter RM: CXC chemokines in cancer angiogenesis and metastases. Adv Cancer Res 106: 91-111, 2010.

20. Joukhadar J, Nevers T and Kalkunte S: New frontiers in reproductive immunology research: Bringing bedside problems to the bench. Exp Rev Clin Immunol 7: 575-577, 2011.
21. Li F, Whyte $\mathrm{N}$ and Niyibizi C: Differentiating multipotent mesenchymal stromal cells generate factors that exert paracrine activities on exogenous MSCs: Implications for paracrine activities in bone regeneration. Biochem Biophys Res Commun 426: 475-479, 2012.

22. Ramasamy R, Tong CK, Yip WK, Vellasamy S, Tan BC and Seow HF: Basic fibroblast growth factor modulates cell cycle of human umbilical cord-derived mesenchymal stem cells. Cell Prolif 45: 132-139, 2012

23. Rose LC, Fitzsimmons R, Lee P, Krawetz R, Rancourt DE and Uludag H: Effect of basic fibroblast growth factor in mouse embryonic stem cell culture and osteogenic differentiation. J Tissue Eng Regen Med 7: 371-382, 2013.

24. Dighe PA, Viswanathan P, Mruthunjaya AK and Seetharam RN: Effect of bFGF on HLA-DR expression of human bone marrow-derived mesenchymal stem cells. J Stem Cells 8: 43-57, 2013.

25. Kim TH, Kim JJ and Kim HW: Basic fibroblast growth factor-loaded, mineralized biopolymer-nanofiber scaffold improves adhesion and proliferation of rat mesenchymal stem cells. Biotechnol Lett 36: 383-390, 2014.

26. Chen L, Jiang W, Huang J, He BC, Zuo GW, Zhang W, Luo Q, Shi Q, Zhang BQ, Wagner ER, et al: Insulin-like growth factor 2 (IGF-2) potentiates BMP-9-induced osteogenic differentiation and bone formation. J Bone Miner Res 25: 2447-2459, 2010.

27. Fukuyo S, Yamaoka K, Sonomoto K, Oshita K, Okada Y, Saito K, Yoshida Y, Kanazawa T, Minami Y and Tanaka Y: IL-6-accelerated calcification by induction of ROR2 in human adipose tissue-derived mesenchymal stem cells is STAT3 dependent. Rheumatology (Oxford) 53: 1282-1290, 2014.

28. Berendsen AD and Olsen BR: How vascular endothelial growth factor-A (VEGF) regulates differentiation of mesenchymal stem cells. J Histochem Cytochem 62: 103-108, 2014.

29. Bai Y, Li P, Yin G, Huang Z, Liao X, Chen X and Yao Y: BMP-2, VEGF and bFGF synergistically promote the osteogenic differentiation of rat bone marrow-derived mesenchymal stem cells. Biotechnol Lett 35: 301-308, 2013.

30. Yuan S, Pan Q, Fu CJ and Bi Z: Effect of growth factors (BMP-4/7 $\&$ bFGF) on proliferation \& osteogenic differentiation of bone marrow stromal cells. Indian J Med Res 138: 104-110, 2013.

31. Hu F, Wang X, Liang G, Lv L, Zhu Y, Sun B and Xiao Z: Effects of epidermal growth factor and basic fibroblast growth factor on the proliferation and osteogenic and neural differentiation of adipose-derived stem cells. Cell Reprogram 15 224-232, 2013

This work is licensed under a Creative Commons Attribution-NonCommercial-NoDerivatives 4.0 International (CC BY-NC-ND 4.0) License. 